VOL. $3(1970), 9-22$.

\title{
Integro-differential equations of Volterra type
}

\section{Rama Mohana Rao and Chris P. Tsokos}

The aim of this paper is concerned with studying the stability properties of an integro-differential system by reducing it into a scalar integro-differential equation. A theorem is stated about the existence of a maximal solution of such systems and a basic result on integro-differential inequalities. Utilizing these results we obtain sufficient conditions for uniform asymptotic stability of the trivial solution of the integro-differential system of the form

$$
x^{\prime}(t)=E(t, x(t), A x), \quad\left({ }^{\prime} \equiv \frac{d}{d t}\right)
$$

where $F \in C\left[R_{+} \times S_{H} \times C(J)\right], A \in C\left[C_{H}, C(J)\right]$ with $C_{H}=\lfloor x \in C(J):\|X\|<H\rfloor, J=0 \leq t \leq a<\infty$, $S_{H}=\left[x \in R^{n}:\|x(t)\|<H, H>0\right.$ for $\left.t \in J\right], C(J)$ denotes the space of continuous functions, $A$ a continuous operator such that $A$ maps $C(J)$ into $C(J)$. The fruitfulness of the results of the paper are illustrated with two applications.

1.

Corduneanu [1], Levin [3] and Nohel [6] among others have studied the stability properties of solutions of integro-differential equations of Volterra type and many interesting results have been accumulated. Quite recently Lakshmikantham and Rama Mohana Rao [2] investigated such a

Received 4 April 1970. The authors thank Professor V. Lakshmikantham for his valuable suggestions during the progress of this research. 
problem by choosing an appropriate minimal class of functions so that it would be possible to estimate the derivative of the Lyapunov function in terms of a scalar function.

Our aim in this paper is to study the stability properties of the integro-differential system by reducing it into a scalar integro-differential equation. In Section 2, we introduce the notation and a lema which is used in the study. In Section 3, we shall develop a theorem about the existence of a maximal solution of integro-differential systems and a basic result on integro-differential inequalities. Applying this theory of integro-differential inequalities, we obtain sufficient conditions for uniform asymptotic stability of the trivial solution of integro-differential systems in Section 4. This study includes the results of Corduneanu [1] as a special case. Two applications are given in Section 5 to illustrate the fruitfulness of our results.

\section{2.}

We shall use the following notations:

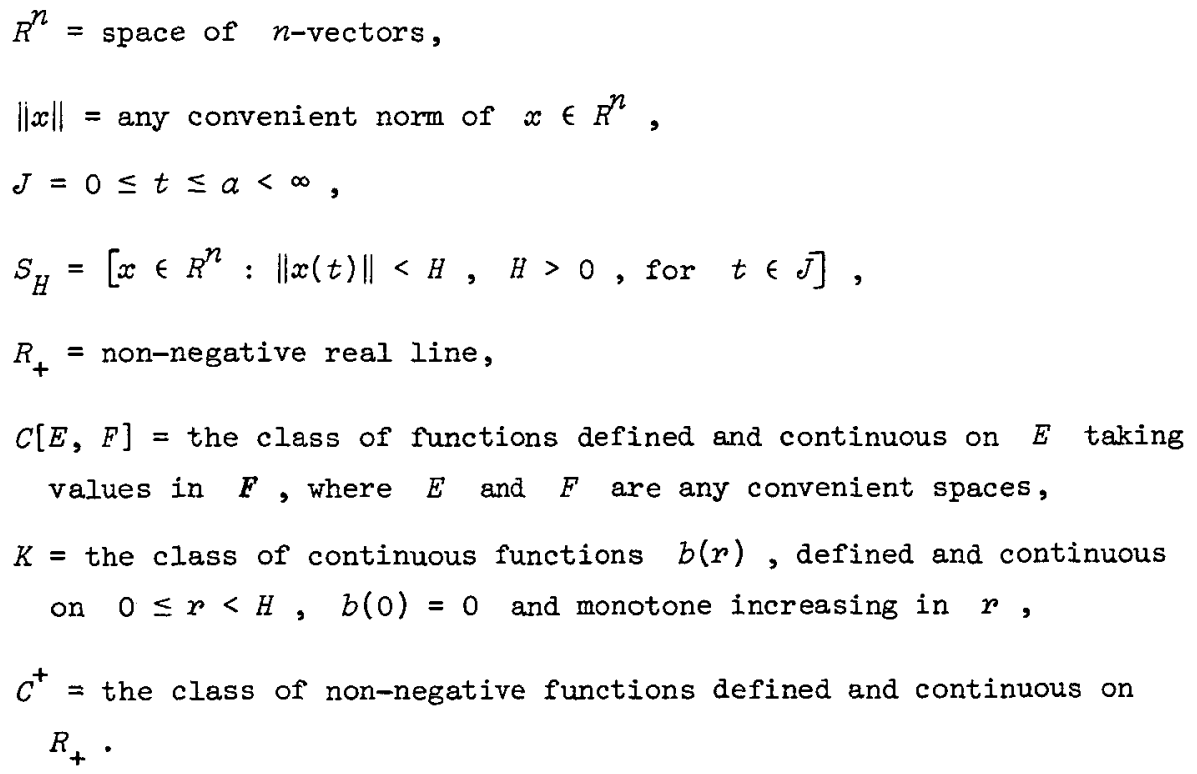

In the presentation of this paper, whenever we employ a vector 
inequality, it is to be understood that the inequality is satisfied componentwise.

Let $E_{1}$ and $F_{1}$ be two partially ordered sets with the partial ordering " $\leq$ " for both sets. We shall assume that the following conditions hold:

$$
\begin{gathered}
x, y, z \in E_{1}, x \leq y, y \leq z \text { implies } x \leq z ; \\
x, y \in E_{1}, x \leq y, y \leq x \text { implies } x=y ; \\
\bar{x}, \bar{y}, \bar{z} \in F_{1}, \bar{x} \leq \bar{y}, \bar{y} \leq \bar{z} \text { implies } \bar{x} \leq \bar{z} ; \\
\bar{x} \in F_{1}, \text { then } \bar{x} \leq \bar{x} .
\end{gathered}
$$

Furthermore, let $P_{1}$ be an operator defined on $E_{1}$ taking its values in $F_{1}$, the function $Q_{1}$ be defined on $E_{1} \times E_{1}$ taking its values in $F_{1}$ and $m$ to denote the maximal solution [5] of equation

$$
P_{1}(x)=Q_{1}(x, x) \text {. }
$$

For $x, y_{1}, y_{2} \in E_{1}$, we shall assume that

$$
y_{1} \leq y_{2} \text { implies } Q_{1}\left(x, y_{1}\right) \leq Q_{1}\left(x, y_{2}\right)
$$

and we define a set $U_{1}$ as follows:

$$
U_{1}=\left[x \in E_{1}: P_{1}(x) \leq Q_{1}(x, x)\right] \text {. }
$$

We now state a lemma about the existence of the maximal solution of equation (2.5), the proof of which follows a similar argument as in [5].

LEMMA 2.1. Let $P_{1}$ and $Q_{1}$ be as defined above. Assume that $Q_{1}$ possesses the property (2.6) and that there exists a function $z_{1}$ defined on $E_{1}$ such that $z_{1}\left(E_{1}\right) \subset E_{1}$ satisfying the conditions

$$
P_{1}\left(z_{1}(y)\right)=Q_{1}\left(z_{1}(y), y\right)
$$

and

$$
P_{1}(x) \leq Q_{1}(x, y)
$$

together imply that

$$
x \leq z_{1}(y) .
$$

Let the set $U_{1}$ defined in (2.7) be non-empty. Then,

$$
z_{1}\left(E_{1}\right) \subset U_{1} \text {. }
$$


Moreover, the existence of $\sup U_{1}$ implies the existence of $\sup _{1}\left(U_{1}\right)$ and vice versa. Also, $\sup U_{1}=\sup z_{1}\left(U_{1}\right)$. Their common value is then the maximal solution of (2.5).

\section{3.}

Let $C(J)$ denote the space of continuous functions $u \in C\left[J, R^{n}\right]$ and $A$ be a continuous operator such that $A$ maps $C(J)$ into $C(J)$. For any two continuous functions $u, v \in C\left[J, R^{n}\right]$ the operator $A$ is assumed to satisfy the following property:

$$
u(t) \leq v(t), \quad 0 \leq t \leq t_{1}, t_{1} \in(0, \infty)
$$

implies

$$
A u \leq A v \text { for } t=t_{1} \text {. }
$$

Let $f \in C\left[J \times R^{n} \times C(J), R^{n}\right]$ and $M$ a constant vector greater than zero such that for $(t, u, v) \in J \times R^{n} \times C(J)$

$$
|f(t, u, v)| \leq M \text {, }
$$

with the function $f$ possessing the quasi-monotone property, that is, for each $i, f_{i}\left(t, u_{1}, u_{2}, \ldots, u_{n}, v_{1}, v_{2}, \ldots, v_{n}\right)$ is monotonic increasing in $u_{1}, u_{2}, \ldots, u_{i-1}, u_{i+1}, \ldots, u_{n}$ and $v_{1}, v_{2}, \ldots, v_{n}$. Now consider the integro-differential system

$$
u^{\prime}(t)=f(t, u(t), A u), u(0)=u_{0} .
$$

THEOREM 3.1. Let $f(t, u, v)$ satisfy the above conditions along with (3.1). Then there exists a maximal solution $m(t)$ of (3.2) on $J$. If $\phi(t)$ is a continuous vector function satisfying the inequalities

$$
\phi(0) \leq m(0)
$$

and

$$
D^{+} \phi(t) \leq f(t, \phi(t), A \phi),
$$

then,

$$
\phi(t) \leq m(t) \text { on } J \text {. }
$$


Proof. Let $E_{1}$ be the set of continuous functions $\psi \in C\left[J, R^{n}\right]$, such that

$$
\psi(t) \leq m(0)+M t, \quad t \in J
$$

For $\psi, \rho \in E_{1}, \psi \leq \rho$ we have

$$
\psi(t) \leq \rho(t), \quad t \in J \text {. }
$$

Similarly, $F_{1}$ is a set of vector-valued functions on $J$ with the components of its elements belonging to $[-\infty, \infty]$ and having the same order relationships as the set $E_{1}$, that is, for $\xi, \eta \in F_{1}, \xi \leq n$ we have

$$
\xi(t) \leq n(t)
$$

From this it is clear that conditions (2.1) to (2.4) hold. Define the operator $P_{1}$ and the function $Q_{1}$ as follows:

$$
P_{1}(\psi)=\left\{D^{+} \psi_{i}(t)\right\}, \quad i=1,2, \ldots, n
$$

and

$$
\begin{array}{r}
Q_{1}(\psi, \rho)=f_{i}\left(t, \rho_{1}(t), \ldots, \rho_{i-1}(t), \psi_{i}(t), \rho_{i+1}(t), \ldots, \rho_{n}(t),\right. \\
\left.A \rho_{1}, A \rho_{2}, \ldots, A \rho_{n}\right) .
\end{array}
$$

It follows from the behavior of $f$ that $Q_{1}$ satisfies (2.6). For $\rho \in E_{1}$, we define

$$
\begin{aligned}
\bar{f}_{i}(t, u)=f_{i}\left(t, \rho_{1}(t), \ldots, \rho_{i-1}(t), u, \rho_{i+1}(t), \ldots, \rho_{n}(t),\right. & \\
& \left.A \rho_{1}, \ldots, A \rho_{n}\right) .
\end{aligned}
$$

Let $\sigma(t)$ be the maximal solution for each $i$ of

$$
u^{\prime}=\bar{f}_{i}(t, u)
$$

such that

$$
\sigma(0)=m(0) \text {. }
$$

The existence of $\sigma(t)$ on $J$ is assured because of the assumptions on $f$. Now, we define a function $z_{1}(\rho)$ such that $z_{1}(\rho(t))=\sigma(t)$. From the basic theorem on differential inequalities it follows that the function $z_{1}(\rho)$ satisfies (2.9) and obviously (2.8) holds. Moreover, 
the set $U_{1}$ is not empty since $m(0)-M t \in U_{1}$ and

$$
\left|\sigma^{\prime}(t)\right| \leq M
$$

Hence, the family of functions $\left\{\sigma_{i}(t)\right\}$ are equi-continuous and uniformly bounded. This proves that $\sup z_{1}\left(U_{1}\right)=\sup \sigma(t)$ is a continuous vector function on $J$. The assertion of the theorem now follows from Lemma 2.1.

\section{4.}

With respect to the objectives of this paper, consider the following integro-differential system

$$
x^{\prime}(t)=F(t, x(t), A x)
$$

where $F \in C\left[R_{+} \times S_{H} \times C(J), R^{n}\right], A \in C\left[C_{H}, C(J)\right]$ with $C_{H}=[x \in C(J):\|x\|<H]$. We shall assume that $F(t, 0,0) \equiv 0$. Let $x(t)=x\left(t, t_{0}, x_{0}\right)$ be any solution of (4.1). In order to avoid repetition we shall only concentrate on uniform asymptotic stability of the trivial solution of the integro-differential system (4.1). For convenience, we shall next define uniform asymptotic stability.

DEFINITION 4.7. The trivial solution of the integro-differential system (4.1) is said to be uniformly asymptotically stable if the following two conditions hold:

(i) for every $\varepsilon>0, t_{0} \in J$, there exists a function $\delta=\delta(\varepsilon)>0$ such that the inequality $\left\|x_{0}\right\| \leq \delta$ implies

$$
\left\|x\left(t, t_{0}, x_{0}\right)\right\|<\varepsilon, \quad t \geq t_{0} ;
$$

(ii) for every $\varepsilon>0, t_{0} \in J$, there exist positive numbers $\delta_{0}$ and $T=T(\varepsilon)$ such that whenever $\left\|x_{0}\right\| \leq \delta_{0}$,

$$
\left\|x\left(t, t_{0}, x_{0}\right)\right\|<\varepsilon, \quad t \geq t_{0}+T
$$

holds.

THEOREM 4.1. Assume that

(i) $g \in C\left[R_{+} \times R_{+} \times R_{+}, R\right], g(t, 0,0) \equiv 0$ and $g(t, u, v)$ is 


$$
\begin{aligned}
& \text { non-decreasing in } v \text { for each }(t, u) \text {; } \\
& \text { (ii) } V \in C\left[R_{+} \times S_{H}, R_{+}\right], \quad V(t, 0) \equiv 0, V(t, x) \text { is Lipschitzian } \\
& \text { in } x \text { for a constant } L=L(H)>0 \text { and for } t \in J, \\
& x(t) \in C(J) \\
& D^{+} V(t, x(t))=\lim _{h \rightarrow 0^{+}} \sup \frac{1}{h}[V(t+h, x(t)+h F(t, x(t), A x)]-V(t, x(t))] \\
& \leq g(t, V(t, x(t)), B V),
\end{aligned}
$$$$
\text { where } B \in C\left[C^{+}, R_{+}\right] \text {; }
$$

(iii) there exists a function $a \in K$ such that for

$$
\begin{aligned}
& (t, x) \in J \times S_{H}, \\
& \quad a(\|x\|) \leq v(t, x) .
\end{aligned}
$$

Then the uniform asymptotic stability of the trivial solution of the scalar equation

$$
r^{\prime}=g(t, r, B r), r\left(t_{0}\right)=r_{0}
$$

implies the uniform asymptotic stability of the trivial solution of the integro-differential system (4.1).

Proof. Suppose that the trivial solution of (4.2) is uniformly asymptotically stable. This implies that it is uniformly stable, Let $0<\varepsilon<H, t_{0} \in J$ be given. Then, given $a(\varepsilon)>0$, there exists a $\delta=\delta(\varepsilon)>0$, such that, whenever $r_{0} \leq \delta$ we have

$$
r\left(t, t_{0}, r_{0}\right)<a(\varepsilon), t \geq t_{0} \text {, }
$$

where $r(t)=r\left(t, t_{0}, r_{0}\right)$ is any solution of (4.2). Let us choose $r_{0}=L\left\|x_{0}\right\|$ so that by assumption $(i i)$ we have $V\left(t_{0}, x_{0}\right) \leq r_{0}$. Now we choose $\delta_{1}(\varepsilon)=\frac{\delta(\varepsilon)}{L}$. Furthermore, we claim that if $\left\|x_{0}\right\| \leq \delta_{1}(\varepsilon)$, we have

$$
\left\|x\left(t, t_{0}, x_{0}\right)\right\|<\varepsilon, \quad t \geq t_{0}
$$


Suppose that this claim is not true, then for $t_{1}>t_{0}$ we have

$$
\left\|x\left(t_{1}, t_{0}, x_{0}\right)\right\|=\varepsilon
$$

and

$$
\left\|x\left(t, t_{0}, x_{0}\right)\right\| \leq \varepsilon, t_{0} \leq t \leq t_{1},
$$

which implies that

$$
a(\varepsilon) \leq V\left(t_{1}, x\left(t_{1}\right)\right)
$$

and

$$
\|x(t)\|<H, \quad t \in\left[t_{0}, t_{1}\right] .
$$

Utilizing the assumption ( $i i)$ and standard computation yields

$$
D^{+} m(t) \leq g(t, m(t), B m), t \in\left[t_{0}, t_{1}\right]
$$

where

$$
m(t)=V(t, x(t)) .
$$

Now, taking $r_{0}$ as defined above and applying Theorem 3.1 we obtain

$$
V(t, x(t)) \leq r(t), \quad t \in\left[t_{0}, t\right] \text {. }
$$

Inequalities (4.3), (4.4) and (4.5) lead to the following contradiction

$$
a(\varepsilon) \leq V\left(t_{1}, x\left(t_{1}\right)\right) \leq r\left(t_{1}\right)<a(\varepsilon) .
$$

Hence, the first condition of Definition 4.1 holds. Now, for $\varepsilon=H$ and $\hat{\delta}_{0}=\delta_{1}(H)$ it follows that

$$
V(t, x(t)) \leq r(t), \quad t \geq t_{0}
$$

Also from the uniform asymptotic stability of the trivial solution of (4.2), we have, given $a(\varepsilon)>0, t_{0} \in J$, there exists a positive number $\delta_{0}$ and $T(\varepsilon)$ such that for $r_{0} \leq \delta_{0}$ it implies that

$$
r\left(t, t_{0}, r_{0}\right)<a(\varepsilon), t \geq t_{0}+T \text {. }
$$

Let $\delta_{0}^{*}=\min \left[\hat{\delta}_{0}, \frac{\delta_{0}}{L}\right]$. Suppose that there exists a sequence $\left\{t_{k}\right\}$, 
$t_{k} \geq t_{0}+T$ with $t_{k} \rightarrow \infty$ as $k \rightarrow \infty$, such that

$$
\left\|x\left(t_{k}, t_{0}, x_{0}\right) i\right\| \varepsilon,
$$

where $x\left(t, t_{0}, x_{0}\right)$ is any solution of (4.1) starting in $\left\|x_{0}\right\| \leq \delta_{0}^{*}$.

Thus in view of assumption ( $i$ ii) of the theorem and inequalities (4.6) and (4.7) we have the following contradiction

$$
a(\varepsilon) \leq v\left(t_{k}, x\left(t_{k}\right)\right) \leq r\left(t_{k}\right)<a(\varepsilon) .
$$

Hence, the second condition of Definition 4.1 holds and the proof is complete.

REMARK. In particular, if

$$
F(t, x, A x)=G(t, x)+\int_{t_{0}}^{t} K(t, s, x(s)) d s,
$$

then equation (4.1) reduces to a perturbed differential system with integral perturbations, that is,

$$
x^{\prime}(t)=G(t, x(t))+\int_{t_{0}}^{t} K(t, s, x(s)) d s .
$$

\section{Applications}

Consider the system of ordinary differential equations,

$$
x^{\prime}=G(t, x)
$$

and integro-differential equations

$$
x^{\prime}(t)=G(t, x(t))+\int_{t_{0}}^{t} K(t, s, x(s)) d s
$$

where $G \in C\left[R_{+} \times S_{H}, R^{n}\right], G(t, x)$ satisfies a Lipschitz condition in $x$ for a constant $\lambda(H)>0$ and $G(t, 0) \equiv 0$. Let $K(t, s, x)$ be defined and continuous on $0 \leq \varepsilon \leq t<\infty,\|x\|<H \leq \infty, K(t, s, 0) \equiv 0$. COROLLARY 5.1. Assume that

(i) the trivial solution of the unperturbed system (5.1) is 
uniformly asymptotically stable;

(ii) $H \in C\left[R_{+} \times R_{+} \times R_{+}, R_{+}\right], H(t, s, 0) \equiv 0, H(t, s, r)$ is nondecreasing in $r$ and $\|K(t, s, x)\| \leq H(t, s,\|x\|), \quad t, s \in J, x \in S_{H} ;$

(iii) for every $d>0$, there exists $a \tau_{d} \geq 0$ and a function $h_{d}(t, s)$ continuous on $\tau_{d} \leq s \leq t<\infty$ such that

$$
H\left(t, s, a^{-1}(r)\right) \leq h_{d}(t, s), a \in K
$$

for all $d \leq r$ and $0 \leq s \leq t$, with

$$
G_{d}(t)=\int_{t}^{t+1}\left\{\int_{t_{0}}^{\xi} h_{d}(\xi, s) d s\right\} d \xi \rightarrow 0 \text { as } t \rightarrow \infty .
$$

Then the trivial solution of the perturbed system (5.2) is uniformly asymptotically stable.

Proof. Since the trivial solution of (5.1) is uniformly asymptotically stable, applying Massera's theorem [4], there exists a Lyapunov function $V(t, x)$ satisfying

$$
\begin{aligned}
& a(\|x\|) \leq V(t, x), \quad a \in K, \\
& |V(t, x)-V(t, y)| \leq L\|x-y\|
\end{aligned}
$$

and

$$
D^{+} V_{(5.1)}(t, x) \leq-C(V(t, x)), \quad C \in K .
$$

From condition ( $i i)$ of Theorem 4.1, inequalities (5.3), (5.4), (5.5), assumption (ii) of the corollary and the monotonic property in $H$, we obtain

$$
D^{+} m(t) \leq-C(m(t))+L \int_{t_{0}}^{t} H\left(t, s, a^{-1}(m(s))\right\} d s
$$

where $m(t)=V(t, x(t))$. Therefore

$$
g(t, r, B r)=-C(r)+L \int_{t_{0}}^{t} H\left(t, s, a^{-1}(r(s))\right) d s .
$$


Now, it remains to verify the uniform asymptotic stability of the trivial solution of

$$
r^{\prime}=g(t, r, B r) \text {. }
$$

This can be done by applying condition (iii) of the corollary and a similar argument as presented in [1]. The solution of equation (5.6) can be written as

$$
r\left(t, t_{0}, r_{0}\right)=r_{0}-\int_{t_{0}}^{t} C(r(s)) d s+L \int_{t_{0}}^{t} \int_{t_{0}}^{\xi} H\left(\xi, s, a^{-I}(r(s))\right) d s d \xi
$$

and for $0<d \leq r(s)$ between $t_{0}$ and $t$, we have

$$
r\left(t, t_{0}, r_{0}\right) \leq r_{0}-\int_{t_{0}}^{t} C(r(s)) d s+L \int_{t_{0}}^{t} \int_{t_{0}}^{\xi} h_{d}(\xi, s) d s d \xi
$$

For $t \geq t_{0} \geq I$ and applying Lemma 3.4 in [7] we obtain

(5.7) $r(t)=r\left(t, t_{0}, r_{0}\right) \leq r_{0}-\int_{t_{0}}^{t} C(r(s)) d s+L \int_{t_{0}-1}^{t} G_{d}(\xi) d \xi$.

Define $Q_{d}(t)=\sup \left[G_{d}(\xi): t-1 \leq \xi<\infty\right]$. Then $Q_{d}(t) \rightarrow 0$ as $t \rightarrow \infty$ and

$$
r(t) \leq r_{0}-C(d)\left[t-t_{0}\right]+L Q_{d}\left(t_{0}\right)\left[t-t_{0}+1\right]
$$

Let $\varepsilon>0$ and choose $\delta=\delta(\varepsilon)$ so that $0<2 \delta<\varepsilon$. Also choose $T_{1}=T_{1}(\varepsilon) \geq \tau_{d}+1$ so that

$$
2 L Q_{\delta}\left(T_{1}\right)<\min [C(\delta), \varepsilon]
$$

Now, for $r_{0} \leq \delta$ and $t_{0} \geq T_{1}$, we claim that

$$
r(t)<\varepsilon \text { for } t_{0} \leq t<\infty \text {. }
$$

Suppose this is not true. Let $T_{3}$ be the first point such that $r\left(T_{3}\right)=\varepsilon$ and let $T_{2}<T_{3}$ be the last point such that $r\left(T_{2}\right)=\delta$. Then $\delta \leq r(t) \leq \varepsilon$ on $\left[T_{2}, T_{3}\right]$, hence by $(5.8)$, 
$\varepsilon=r\left(T_{3}\right) \leq\left[L Q_{\delta}\left(T_{2}\right)-C(\delta)\right]\left[T_{3}-T_{2}\right]+L Q_{d}\left(T_{2}\right)+r_{0} \leq L Q_{d}\left(T_{1}\right)+r_{0}$

$$
<\frac{\varepsilon}{2}+\frac{\varepsilon}{2}=\varepsilon \text {, }
$$

a contradiction, proving (5.10). This proves the uniform stability of the trivial solution of $(5.6)$. For the rest of the proof, choose $\delta_{0}=\delta(\varepsilon)$, $T_{0}=T_{1}(\varepsilon)$. Fix $t_{0} \geq T_{0}$ and $r_{0} \leq \delta_{0}$. Then (5.10) implies that

$$
r\left(t, t_{0}, r_{0}\right)<\varepsilon \text { on }\left[t_{0}, \infty\right) \text {. }
$$

For $n>0$, choose $\delta(n)$ and $T_{1}(n)$ as before so that (5.9) holds. Choose

$$
T=\left[C(\delta) T_{1}(\eta)+2 L Q_{\delta}(1)+2 \delta\right][C(\delta)]^{-1} \geq T_{1}(\eta)
$$

which does not depend on $t_{0}$ or $r_{0}$. We now claim that (5.11) $\quad r\left(t_{1}, t_{0}, r_{0}\right)<\delta$ for some $t_{1}$ in $\left[t_{0}+T_{1}, t_{0}+T\right]$.

Suppose that our claim is false, then

$$
r\left(t_{1}, t_{0}, r_{0}\right) \geq \delta \text { on }\left[t_{0}+T_{1}, t_{0}+T\right] \text {. }
$$

Let $y_{0}=r\left(t_{0}+T_{1}, t_{0}, r_{0}\right)$. Then

$0<\delta \leq r\left(t_{0}+T, t_{0}+T_{1}, y_{0}\right) \leq\left[L Q_{\delta}\left(t_{0}+T_{1}\right)-C(\delta)\right]\left[T-T_{1}\right]+L Q_{\delta}\left(t_{0}+T_{1}\right)+y_{0}$

$$
\leq-\frac{C(\delta)}{2}\left[T-T_{1}\right]+L Q_{\delta}(1)+\delta=0 \text {, }
$$

a contradiction, proving (5.11). Thus by (5.10)

$$
r\left(t, t_{1}, r\left(t_{1}, t_{0}, r_{0}\right)\right)<n \text {, on }\left[t_{1}, \infty\right] \text {, }
$$

since $t_{1} \geq t_{0}+T_{1} \geq T_{1}$ and $r\left(t_{1}, t_{0}, r_{0}\right)<\delta$. Hence

$$
r\left(t, t_{0}, r_{0}\right)<n \text { for } t \geq t_{0}+r \text {. }
$$

Since $\eta$ is arbitrary $r\left(t, t_{0}, r_{0}\right) \rightarrow 0$ as $t \rightarrow \infty$. Also as $T$ depends only on $n$ and $\delta$ depends only on $\varepsilon$, the trivial solution of (5.6) is uniformly asymptotically stable. Consequently by Theorem 4.I the stated result follows.

\section{COROLLARY 5.2. Assume that}


(i) the trivial solution of the unperturbed system (5.1) is exponentially stable;

(ii) the function $K(t, s, x)$ is defined and continuous on $0 \leq s \leq t<\infty,\|x\|<H \leq \infty$

$$
\|K(t, s, x)\| \leq K_{1}(t, s) w(s,\|x\|)
$$

where $K_{1}(t, s) \geq 0$ is defined and continuous on $0 \leq s \leq t<\infty, \quad w(t, r) \in C\left|R_{+} \times R_{+}, R\right|, \quad w(t, 0) \equiv 0$, satisfies a Lipschitz condition in $r$ and non-decreasing in $r$;

(iii) there exists a function $\tilde{h}(t, s)$ defined and continuous on $0 \leq s \leq t<\infty$ and satisfying the inequality

$$
\tilde{h}(t, s) \leq M_{1} \exp \left[-\lambda_{1}(t-s)\right] \text {, for } 0 \leq s \leq t \text {, }
$$

$\lambda_{1}, M_{1}>0$, where

$$
\tilde{h}(t, s)=\int_{s}^{t} K_{1}(\tau, s) \exp \left[-\lambda_{1}(t-\tau)\right] d \tau .
$$

Then the trivial solution of the perturbed system (5.2) is exponentially stable.

Proof. Since the trivial solution of (5.1) is exponentially stable, there exists a Lyapunov function $V(t, x)$ satisfying the following:

(i) $\|x\| \leq V(t, x)$;

(ii) $|V(t, x)-V(t, y)| \leq L\|x-y\|$;

(iii) $D^{+} V_{(5.1)}(t, x) \leq-\lambda V(t, x), \lambda>0$.

It is easy to show that

$$
g(t, r, B r)=-\lambda r+L \int_{0}^{t} K_{1}(t, s) \omega(s, r(s)) d s .
$$

Now, by using similar arguments as in [1], it is easy to show that the trivial solution of (5.6) is exponentially stable and applying Theorem 4.1 we obtain the desired result. 


\section{References}

[1] C. Corduneanu, "Quelques problèmes qualitatifs de la theorie des équations intégro-différentielles", Colzoq. Math. 18 (1967), $77-87$.

[2] V. Lakshmikantham and M. Rama Mohana Rao, Integro-differential equations and extension of Lyopunov's method (URI Tech. Report no. 7, February 1969).

[3] J.J. Levin, "The qualitative behavior of a nonlinear Volterra equation", Proc. Amer. Math. Soc. 16 (1965), 711-718.

[4] José L. Massera, "Contribution to stability theory", Ann. of Math. (2) $64(1956), 182-206$.

[5] W. Mlak, "Note on maximal solutions of differential equations", Contributions to Differential Equations 1 (1963), 461-465.

[6] John A. Nohel, "Remarks on nonlinear Volterra equations", Proc. U.S. - Japan Seminar on Differential and Eunctional Equations (Minneapolis, Minn., 1967), 249-266. (Benjamin, New York, 1967).

[7] Aaron Strauss and James A. Yorke, "Perturbation theorems for ordinary differential equations", J. Differential Equations 3 (1967), 15-30.

Indian Institute of Technology Kanpur,

Kanpur, India;

Virginia Polytechnic Institute and State University, Blacksburg, Virginia, USA. 